\title{
Role and Application of Diffusion-weighted Imaging in Evaluation of Prostate Cancer
}

\begin{abstract}
Introduction: Diffusion-weighted imaging (DWI) is an important part of magnetic resonance imaging (MRI) in the evaluation of specific organs, including the breast, kidney, liver, and prostate. Prostate cancer lesions are composed of tightly packed cells with reduced extracellular space, which can be visualized on DWIs as areas of restricted diffusion (i.e., high-signal intensity), with corresponding low-signal intensity on apparent diffusion coefficient (ADC) maps and low mean ADC value. Objective: The objective of this study is to identify the appropriate cutoff and mean ADC value to diagnose neoplastic prostatic lesions in central India. Materials and Methods: Sixty-six patients with suspected prostatic pathology were included in this study. All patients underwent MRI on a 1.5-T scanner with a phased-array body coil. MRIs were evaluated compared with the histopathological staging. Results: The diagnostic accuracy of DWI in predicting malignancy was $39 / 41$, i.e., $95.12 \%$, which is the positive predicted value. The mean ADC for benign category was 1.14 with standard deviation (SD) of 0.14 while mean for prostatitis was 0.91 with SD of 0.26 and for carcinoma was 0.75 with SD of 0.19 . The difference in the means was statistically highly significant. Conclusion: DWI demonstrates the restriction of diffusion and the reduction of ADC values in neoplastic tissue and improves the diagnostic accuracy in lesion characterization. This technique allows short acquisition time and provides high-contrast resolution between neoplastic and normal tissue. This technique can be a useful adjunct to the established dynamic contrast-enhanced-MRI.
\end{abstract}

Keywords: Apparent diffusion coefficient, diffusion-weighted imaging, prostate cancer, prostate pathologies

\section{Introduction}

The application of a diffusion-weighted (DW) sequence in whole-body imaging has gained more popularity and improved the diagnostic accuracy in lesion characterization with new technical developments in magnetic resonance imaging (MRI), including echo planar imaging (EPI), and stronger gradients ${ }^{[1]}$ leading to a reduction in the amount of time required for DW imaging (DWI) to $<1 \mathrm{~min}$. Therefore, these sequences can be added to the imaging protocol without significantly increasing overall acquisition time. Another benefit of DWI is its use of inherent tissue contrast; hence, no exogenous contrast material is required.

\section{Principle of diffusion-weighted imaging}

DWI exploits the property of constant Brownian motion of the water molecules in tissue. This property is affected by increased cellularity, tissue organization, extracellular

This is an open access journal, and articles are distributed under the terms of the Creative Commons Attribution-Non Commercial-ShareAlike 4.0 License, which allows others to remix, tweak, and build upon the work non-commercially, as long as appropriate credit is given and the new creations are licensed under the identical terms.

For reprints contact: reprints@medknow.com space, and integrity of cell membranes. DWI measures the Brownian motion of water molecules in tissues while increased cellularity restricts water diffusion. Apparent diffusion coefficient (ADC) derived from DWI demonstrates reduced ADC values and increased fractional anisotropy in prostate cancer. Free water molecules are in constant random motion, known as Brownian motion, which is related to thermal kinetic energy. In contrast, the motion of water molecules within the cellular microenvironment is impeded by their interaction with cellular compartments, including the cell wall and intracellular organelles. ${ }^{[2]}$ In other words, restriction in the diffusion of water molecules is directly proportional to the degree of cellularity of the tissue. Sato et al. ${ }^{[3]}$ compared the ADC values of normal and cancerous tissue in both the peripheral and transition zones at $1.5 \mathrm{~T}$. In both zones, the ADC values of prostate cancer were significantly lower than those of benign tissue. ${ }^{[3]}$

How to cite this article: Lahoti AM, Lakhotiya AR, Ingole SM, Dhok AP, Mudaliar PN. Role and application of diffusion-weighted imaging in evaluation of prostate cancer. Indian J Med Paediatr Oncol 2018;39:349-54.

\section{Amol Madanlal \\ Lahoti, \\ Ankita Ramprasad \\ Lakhotiya ${ }^{1}$, Sarang Manohar Ingole ${ }^{2}$, \\ Avinash Parshuram Dhok, Prashant N Mudaliar}

Department of Radiology, NKP Sims and Lata Mangeshkar Hospital, Nagpur, 'Department of Ophthalmology, Laxmi Eye Institute, Panvel, ${ }^{2}$ Department of Radiology, Seth GS Medical College and KEM Hospital, Mumbai, Maharashtra, India

Address for correspondence: Prof. Avinash Parshuram Dhok, Department of Radiology, NKP Sims and Lata Mangeshkar Hospital, Nagpur, Maharashtra, India.

E-mail: amollahoti20031987@ gmail.com

Access this article online

Website: www.ijmpo.org

DOI: 10.4103/ijmpo.ijmpo_41_17 Quick Response Code:

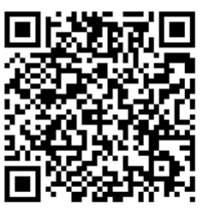


This restricted diffusion is observed primarily in malignancies, [Figures 1-5] hypercellular metastases, and fibrosis, which contain a greater number of cells with intact cell walls than healthy tissue. Cancer tissue has higher signal intensity on high-b-value DWIs than healthy prostate tissue and will demonstrate decreased ADC values. ${ }^{[3-6]}$

Quantitative analysis with diffusion-weighted imaging-apparent diffusion coefficient mapping

$\mathrm{ADC}$ is calculated during post processing with use of at least two different $b$ values. A more accurate ADC value can be obtained using more DWIs with different $b$ values. The final image with different ADC values calculated for each pixel of an image is referred to as an ADC map. The regions with more restricted diffusion and therefore higher DW signal show lower ADC values.

\section{Artifacts and pitfalls of diffusion-weighted imaging}

\section{T2 Shine-through effect}

The SE sequence used in DWI is T2 weighted, and the signal intensity of the tissue depends on both the T2 signal as well as the degree of signal attenuation after applying the motion-probing gradient pulses. In tissues with very long $\mathrm{T} 2$ relaxation times, the strong T2signal may be mistaken for restricted diffusion. This phenomenon is known as T2 shine-through effect. $\mathrm{V}$ can differentiate the T2 shine through and true diffusion restriction by ADC mapping and ADC value.An area of low signal intensity on ADC (low $\mathrm{ADC}$ value)is seen in the true diffusion restriction and false restriction/shine through is seen as area of high signal intensity. ${ }^{[7]}$

\section{Susceptibility artifact}

Other sources of susceptibility artifact include medical devices (eg, metallic stents, surgical clips) and grafts. One way to reduce susceptibility artifact is to shorten the echo time and increase the bandwidth with image acquisition. ${ }^{[8]}$

\section{Motion artifact}

Motion artifacts due to moving organs are a source of image degradation and artifacts in DWI. Motion artifacts are more pronounced along the phase-encoding direction, where it creates "ghost" images. Increasing the speed of image acquisition, with single-shot EPI and using parallel imaging are possible ways to decrease this artifact. ${ }^{[9]}$

\section{Materials and Methods}

A diagnostic study to evaluate the role of DWI and $\mathrm{ADC}$ value in various prostatic pathologies, with main focus on detecting prostate cancer. Total 66 patients were informed about the nature, objective of the study, and written informed consent were taken, and Institutional Ethics Committee approval was taken beforehand. Patients with clinical suspicion of Prostate pathologies (lower urinary tract symptoms such as increased frequency of micturition, hesitancy, urgency, and hard/enlarged prostate on digital rectal examination), enlarged prostate on ultrasound abdomen and/or raised prostate-specific antigen (PSA) levels $(>4 \mathrm{ng} / \mathrm{ml})$ were included in this study. All MRI examinations were performed with a $1.5-\mathrm{T}$ using the multichannel body matrix phased-array coil. The examinations were performed in T1-WI, T2-WI, DWI, and T1-weighted sequences. ADCs were calculated from transverse DWI obtained using a single-shot echo-planar imaging sequence with the following parameters: TR/TE: $3400 / 75 \mathrm{~ms}$ and $b$ values of $0,100,300,800$, and $1000 \mathrm{~s} / \mathrm{mm}$.

Patient unwilling or unable (claustrophobic) to undergo MRI/magnetic resonance spectroscopy (MRS), Metallic hip implant or any other metallic implant or device that might distort local magnetic field and compromise the quality of MRI/MRS and recent biopsy were excluded from the study.

Machine used

MRI-1.5 Tesla from GE health care with body coil.

\section{Statistical analysis}

Continuous variables were summarized as mean and standard deviations (SDs) whereas nominal/categorical variables as proportions (\%). A Chi-square test was used for the analysis of nominal/categorical variables. Z-test for difference between two proportions was used for comparison of nominal/categorical variables. Unpaired " $\mathrm{t}$ " test and ANOVA test were used for continuous variables. Diagnostic accuracy of MRI was assessed by means of sensitivity, specificity, positive predictive value, and negative predictive value. $P<0.05$ was taken as significant. Medcalc 14.0.0 version was used for all statistical calculations.

\section{Results}

In our study, majority of patients belonged to the age group 60-69 years constituting about 45.4\%. Mean age of patients with benign pathology in our study was $60.68 \pm 8.69$ and with malignant pathology is $68.97 \pm 7.97$ years. These findings are in concordance with previous studies by Gann ${ }^{[10]}$ Squillaci et al., ${ }^{[17]}$ and Costouros et al..$^{[18]}$

Table 1 gives the descriptive statistics such as mean and SD for ADC. The comparison of mean ADC was performed for each MRI-based zone across histological interpretations. In the peripheral zone (PZ), the mean ADC across interpretation groups was statistically significant with $P$ value of $0.0211(P<0.05)$. In addition, in the transition zone, the mean ADC across interpretation groups differed highly significantly with $P<0.0001$ as per one-way ANOVA. In other two zones, the analysis could not be performed due to inadequacy of sample size. 


\begin{tabular}{|c|c|c|c|c|}
\hline \multirow[t]{3}{*}{ MRI based zones } & \multirow{2}{*}{\multicolumn{3}{|c|}{$\begin{array}{c}\text { Mean } \pm \text { SD (n) } \\
\text { Histopathology }\end{array}$}} & \multirow[t]{3}{*}{$P^{*}$} \\
\hline & & & & \\
\hline & Benign prostatic hyperplasia & Carcinoma & Prostatitis & \\
\hline Central & $1.02 \pm 0.00$ & $0.74 \pm 0.17(3)$ & $1.10 \pm 0.00(1)$ & - \\
\hline Peripheral & $1.07 \pm 0.23(5)$ & $0.78 \pm 0.19(21)$ & $0.82 \pm 0.20$ (4) & $0.0211(\mathrm{~S})$ \\
\hline Transition & $1.18 \pm 0.09(13)$ & $0.72 \pm 0.21(14)$ & $0.66 \pm 0.00$ & $<0.0001$ (HS) \\
\hline Peripheral and transition & 0.00 & $0.62 \pm 0.05(3)$ & 0.00 & - \\
\hline
\end{tabular}

*Obtained using ANOVA for peripheral, $t$-test for transition: S - Significant; HS - Highly significant. SD - Standard deviation;

MRI - Magnetic resonance imaging

Comparison of ADC across grades based on Gleason score is presented in Table 2. It is found that with increase in Gleason grade, the mean, and median ADC value decreases. Table 2 gives the descriptive statistics such as mean, SD, and median for ADC. There were 9 cases with low grade having mean $\mathrm{ADC}$ of 0.872 and $\mathrm{SD}$ of 0.123 while 14 cases of intermediate grade with mean ADC of 0.719 and SD of 0.121 and 17 cases of high grade with mean ADC of 0.682 and SD of 0.212. There were 26 cases of without gradation. The difference in the mean ADC values across three grades was statistically significant with $P$ value of $0.0284(P<0.05)$ using one-way ANOVA.

Comparison of mean ADC across different types of prostatic lesions is presented in Table 3. Table 3 gives the comparison of mean $\mathrm{ADC}$ across difference prostatic lesions. The mean ADC for benign category was 1.14 with SD of 0.14 while mean for prostatitis was 0.91 with SD of 0.26 and for carcinoma was 0.75 with SD of 0.19 . The difference in the means was statistically highly significant with $P<0.0001$ using one-way ANOVA.

Comparison of diffusion-weighted imaging and histopathology finding in predicting malignancy is presented in Table 4. Table 4 gives the comparison of diffusion-weighted imaging with histopathological finding in predicting malignancy. There were 39 cases correctly diagnosed as malignant while 17 cases were correctly diagnosed as benign (true negative). There were 8 cases diagnosed as nonmalignant on histopathology but malignant on DWI, and 2 cases were diagnosed as malignant on histopathology but nonmalignant on final diagnosis, after combining all modalities.

Accordingly, the diagnostic accuracy of DWI in predicting malignancy was $39 / 41$, i.e., $95.12 \%$, which is the positive predicted value.

\section{Discussion}

\section{Prostate cancer screening}

All men who are 50-60 years or older presenting with lower urinary tract symptoms are now offered serum PSA testing. The diagnostic evaluation is further offered by transabdominal or transrectal ultrasound.
Anatomy of prostate gland

Figure 1 depicts prostate zonal anatomy.

\section{Magnetic resonance imaging}

Prostate is an organ in which various focal or diffuse, benign or malignant primary pathology/lesion can be found. Prostatic pathology/focal masses are diagnosed using ultrasonography (USG), and it is screening modality of choice. If needed, then further TRUS and TRUS-guided biopsy is done to get more accurate diagnosis. Most of the lesion can be diagnosed using transabdominal or transrectal ultrasound.

Magnetic resonance imaging (MRI) is imaging modality, which adds to the characterization about anatomy, pathology, and extent of pathologies detected on USG (ultrasound study). MRI has many advantages (e.g., high-contrast resolution, ability to obtain images in any plane, i.e., multiplanar imaging, no ionizing radiation, and safety of using particulate contrast media rather than those containing iodine) that make it a favorable modality.

In our study, lesions were identified and diagnosed using T2W, DWI, and MRS sequences. Lesions were localized to a particular zone.

\section{Diffusion-weighted imaging}

DWI exploits the property of constant Brownian motion of the water molecules in tissue. This property is affected by increased cellularity, tissue organization, extracellular space, and integrity of cell membranes. Prostate cancer foci are composed of tightly packed cellular elements with reduced extracellular space, which can be visualized on DWI images as areas of restricted diffusion (high signal intensity), with corresponding low-signal intensity on ADC maps. Diffusion-weighted imaging demonstrates the restriction of diffusion and the reduction of ADC values in neoplastic tissue. This technique allows short acquisition time and provides high-contrast resolution between neoplastic and normal tissue.

Tanimoto et al. evaluate the clinical value of DWI and dynamic MRI in combination with T2-weighted imaging (T2W) for the detection of prostate cancer 83 patients with elevated serum prostate specific 


\begin{tabular}{|c|c|c|c|}
\hline Grade & $n$ & $\operatorname{Mean} \pm$ SD & Median \\
\hline Low & 9 & $0.872 \pm 0.123$ & 0.895 \\
\hline Intermediate & 14 & $0.719 \pm 0.121$ & 0.718 \\
\hline High & 17 & $0.682 \pm 0.212$ & 0.661 \\
\hline
\end{tabular}

$P=0.0284$ (significant) using one-way ANOVA. SD - Standard deviation

Table 3: Comparison of mean apparent diffusion coefficient across different prostatic lesions

\begin{tabular}{lcc}
\hline Histopathology & $\boldsymbol{n}$ & Mean \pm SD \\
\hline BPH & 19 & $1.14 \pm 0.14$ \\
Prostatitis & 6 & $0.91 \pm 0.26$ \\
Carcinoma & 41 & $0.75 \pm 0.19$ \\
\hline
\end{tabular}

$P<0.0001$ using one-way ANOVA. BPH - Benign prostatic

hyperplasia; SD - Standard deviation

\begin{tabular}{|c|c|c|c|}
\hline \multirow[t]{2}{*}{ Histopathology } & \multicolumn{2}{|c|}{ DWI } & \multirow{2}{*}{$P^{*}$} \\
\hline & Noncarcinoma & Carcinoma & \\
\hline Benign & 17 & 8 & $<0.0001$ \\
\hline Malignant & 2 & 39 & (HS) \\
\hline Sensitivity & $82.98 \%$ & & \\
\hline Specificity & $89.47 \%$ & & \\
\hline Positive predictive value & $95.12 \%$ & & \\
\hline Negative predictive value & $68.00 \%$ & & \\
\hline
\end{tabular}

*Obtained using Chi-square test. HS - Highly significant;

DWI - Diffusion-weighted imaging

antigen (PSA) levels ( $>4.0 \mathrm{ng} / \mathrm{mL}$ ) were evaluated by T2W, DWI, and dynamic MRI at $1.5 \mathrm{~T}$ before needle biopsy. The data from the results of the $\mathrm{T} 2 \mathrm{~W}$ alone (protocol $\mathrm{A}$ ), combination of $\mathrm{T} 2 \mathrm{~W}$ and DWI (protocol B), and the combination of T2W+DWI and dynamic MRI (protocol C) was entered into a receiver operating characteristic (ROC) curve analysis, under the results of systemic biopsy as the standard of reference. Prostate cancer was pathologically detected in 44 of the 83 patients. The sensitivity, specificity, accuracy, and the area under the ROC curve (Az) for the detection of prostate cancer were as follows: $73 \%, 54 \%$, $64 \%$, and 0.711 , respectively, in protocol A; $84 \%, 85 \%$, $84 \%$, and 0.905 , respectively, in protocol $\mathrm{B}$; and $95 \%$, $74 \%, 86 \%$, and 0.966 , respectively, in protocol C. The sensitivity, specificity, and accuracy were significantly different between the three protocols $(P<0.01)$. In patients with elevated serum PSA levels, the combination of T2W, DWI, and dynamic MRI may be a valuable tool for detecting prostate cancer and avoiding an unnecessary biopsy without missing prostate cancer. ${ }^{[11]}$

Haider et al. (2007) et al. Combined T2-weighted and DW MRI for localization of prostate cancer T2-weighted imaging and DWI ( $b$ value $=600 \mathrm{~s} / \mathrm{mm} 2$ ) were performed in 49 patients before radical prostatectomy using an endorectal

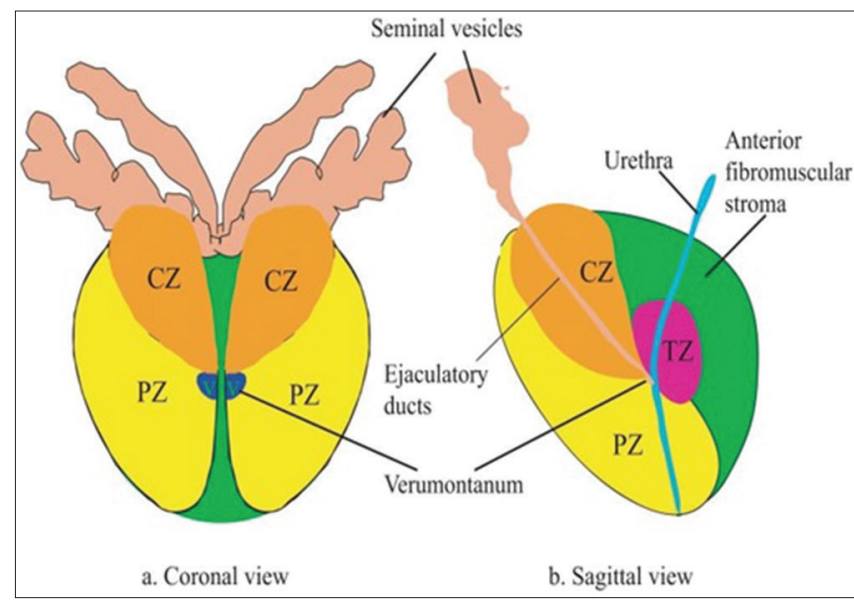

Figure 1: Prostate-Zonal anatomy (Abbreviations: CZ - Central zone; TZ - Transition zone; PZ - Peripheral zone)
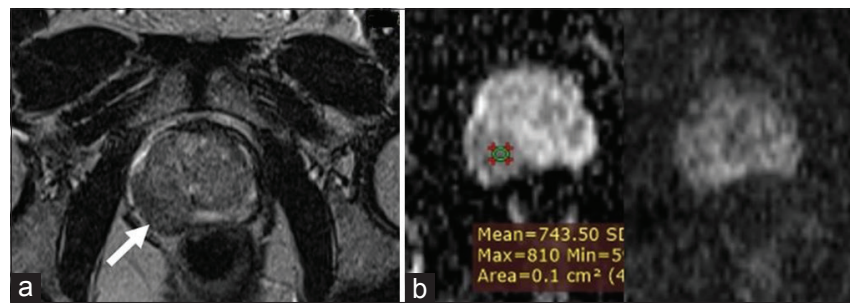

Figure 2: Peripheral Zone Carcinoma (a) Right peripheral zone hypointensity which is equivocal (b) Focal mildly hypointense on apparent diffusion coefficient and mildly hyperintense on high b-value diffusion-weighted imaging

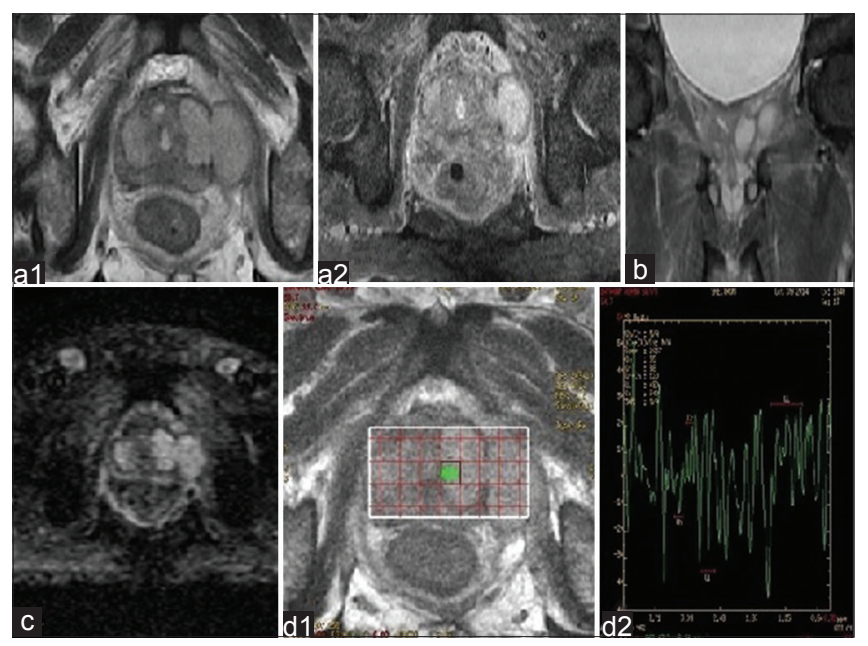

Figure 3: Peripheral zone carcinoma. (a1 and a2) Axial image showing iso to hypointensity involving predominantly left peripheral and transition zone with extracapsular extension. (b) Coronal image showing extracapsular extension of the mass lesion. (c) Area of restricted diffusion with extracapsular extension (d1 and d2) MRS shows elevated choline and reduced citrate peak suggestive of prostatic carcinoma

coil at $1.5 \mathrm{~T}$ in this prospective trial. The $\mathrm{PZ}$ of the prostate was divided into sextants and the transition zone into the left and right halves. Only tumors with an area of more than $0.13 \mathrm{~cm}^{2}(>4 \mathrm{~mm}$ diameter) and a Gleason score of $>$ or $=6$ were considered significant. The area under the ROC curve (A (z)) was used to the assess accuracy. In the PZ, the 

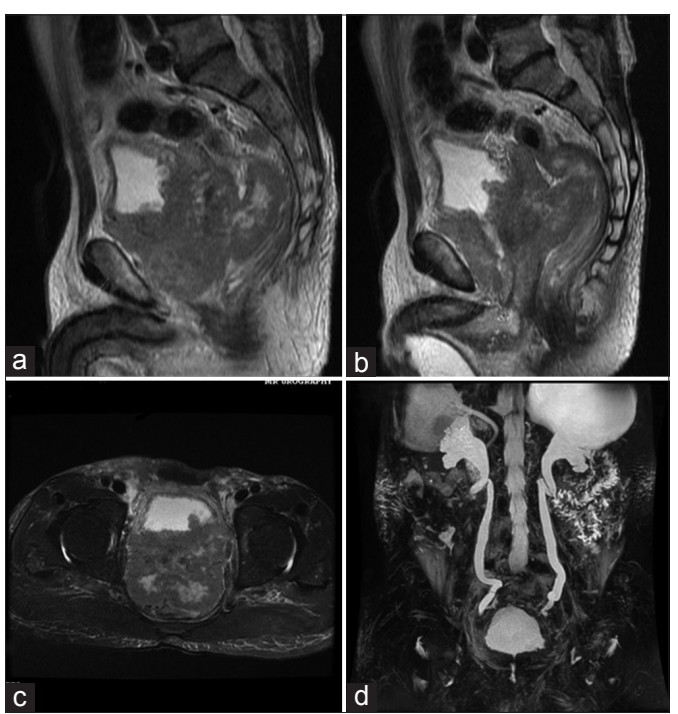

Figure 4: MRI Urography study of Diffuse Prostatic Carcinoma showing, ( $a$ and $b$ ) sagittal T2WI images, large prostatic mass that is bulging into the urinary bladder base and pushing the large bowel loops more posteriorly with loss of fat planes between prostate and adjacent organs, (c) axial T2WI showing heterogenous isodence to hyperintense mass lesion protruding into bladder. (d) 3D MR Urography image showing hydronephrosis caused by the bladder outlet obstruction because of prostatic mass

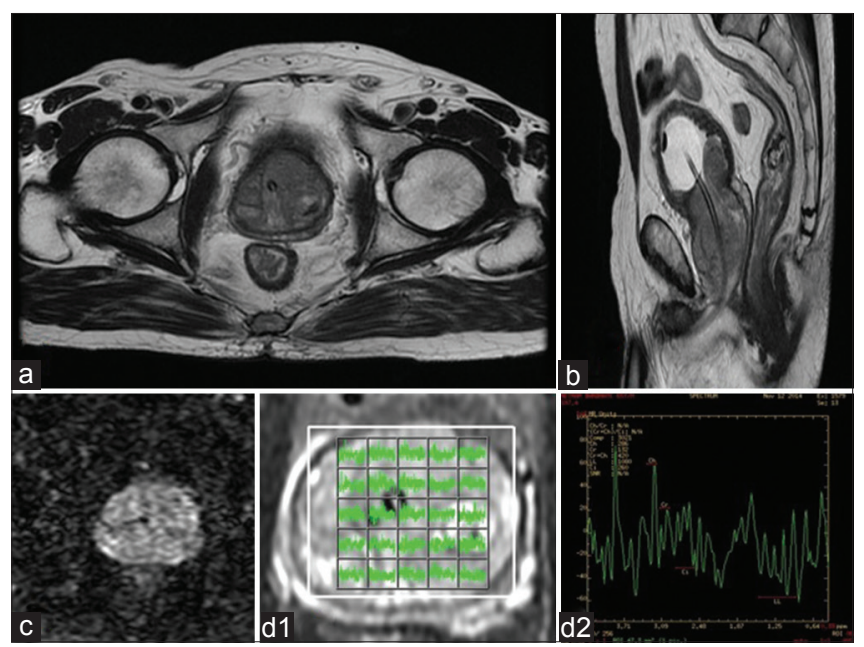

Figure 5: Transition zone Carcinoma. (a) T2W axial image showing ill-defined hypointensity involving predominantly left transition zone. (b) Sagittal image of the same. (c) Focal area of restricted diffusion. (d) MRS shows elevated choline and reduced citrate peak

A (z) value was significantly higher $(P=0.004)$ for T2 plus DWI $(\mathrm{A}(\mathrm{z})=0.89)$ than for T2 imaging alone $(\mathrm{A}(\mathrm{z})=0.81)$. The performance was poorer in the transition zone for both T2 plus DWI $(\mathrm{A}(\mathrm{z})=0.78)$ and $\mathrm{T} 2(\mathrm{~A}(\mathrm{z})=0.79)$. For the whole prostate, sensitivity was significantly higher $(P<0.001)$ with T2 plus DWI $(81 \%$ [120/149]) than with T2 imaging alone (54\% [81/149]), with T2 plus DWI showing only a slight loss in specificity compared with T2 imaging alone (84\% [204/243] vs. 91\% [222/243], respectively). Combined T2 and DWI MRI are better than T2 imaging alone in the detection of significant cancer (Gleason score $\geq 6$ and diameter $>4 \mathrm{~mm}$ ) within the $\mathrm{PZ}$ of the prostate. ${ }^{[12]}$
Kozlowski et al. DW MRI at $1.5 \mathrm{~T}$ were carried out in two groups of patients. MRI data were correlated with the biopsy and histopathology. The performance of two sequences - a single-shot fast spin echo (FSE) (14 patients) and a single-shot EPI (15 patients) - was compared. Average $\mathrm{ADC}$ values from the normal PZ, central gland (CG), and the tumor were calculated from $b$ values of 0 and 600 . Tukey-Kramer test was used for statistical analysis. EPI produced higher values of ADC $\left(10 \_3 \mathrm{~mm}^{2} / \mathrm{s}\right)$ than FSE sequence: $1.992 \mathrm{~F} 0.208$ vs. $1.573 \mathrm{~F} 0.270$ in $\mathrm{PZ}(\mathrm{P} \mathrm{b} .001)$, $1.518 \mathrm{~F} 0.126$ vs. $1.373 \mathrm{~F} 0.179$ in $\mathrm{CG}$, and $1.214 \mathrm{~F} 0.254$ vs. $0.993 \mathrm{~F} 0.158$ in PCa (P b. 01). In conclusion, both EPI and FSE sequences showed differences in ADC between normal $\mathrm{PZ}, \mathrm{CG}$, and $\mathrm{PCa}$; however, EPI produced significantly higher ADC values than FSE. ${ }^{[13]}$

Differences in ADC values between patients with low grade, intermediate grade, and highly malignant grade disease $(P=0.000)$ are consistent with the literature data available, which states that higher Gleason score is associated with lower ADC, probably due to specific infiltrative growth of prostate tumors. Such an organization is opposite to the glandular organization of more well-differentiated prostate cancer, which is similar to healthy prostate. ${ }^{[14,15]}$

There are many publications related to the significance of DWI and ADC in prostate cancer diagnostics. The literature reports on importance of DWI/ADC in prostate cancer diagnostics, especially in case where difficulties are encountered with classical imaging aimed at tumor localization within the PZ.

Abscess also shows restriction on diffusion just like most prostate cancer. However, it appears hyperintense on T2WI, and it show peripheral enhancement only instead of heterogenous or diffuse enhancement of neoplastic tissue. ${ }^{[16]}$

\section{Conclusion}

The present study was undertaken to determine the role of DWI in the evaluation of prostatic pathologies, mainly prostate neoplasm and to determine the mean ADC values for different prostatic pathologies. MRI scans were reviewed, and the prostatic lesions were identified and characterized and the results were correlated with histopathological findings. DWI with ADC mapping and ADC values found to be very useful adjuvant in characterization of prostatic pathologies. We recommend that DWI should be included in the routine MRI protocol for evaluation of prostate pathologies.

Financial support and sponsorship

Nil.

Conflicts of interest

There are no conflicts of interest. 


\section{References}

1. Koh DM, Collins DJ. Diffusion-weighted MRI in the body: Applications and challenges in oncology. AJR Am J Roentgenol 2007;188:1622-35.

2. Qayyum A. Diffusion-weighted imaging in the abdomen and pelvis: Concepts and applications. Radiographics 2009;29:1797-810.

3. Sato C, Naganawa S, Nakamura T, Kumada H, Miura $\mathrm{S}$ Differentiation of noncancerous tissue and cancer lesions by apparent diffusion coefficient values in transition and peripheral zones of the prostate. J Magn Reson Imaging 2005;21:258-62.

4. Van As N, Charles-Edwards E, Jackson A, Jhavar S, Reinsberg S, Desouza N, et al. Correlation of diffusion-weighted MRI with whole mount radical prostatectomy specimens. $\mathrm{Br} \mathrm{J}$ Radiol 2008;81:456-62.

5. Issa B. In vivo measurement of the apparent diffusion coefficient in normal and malignant prostatic tissues using echo-planar imaging. J Magn Reson Imaging 2002;16:196-200.

6. Shimofusa R, Fujimoto H, Akamata H, Motoori K, Yamamoto S, Ueda $\mathrm{T}$, et al. Diffusion-weighted imaging of prostate cancer. J Comput Assist Tomogr 2005;29:149-53.

7. Stadnik TW, Demaerel P, Luypaert RR, Chaskis C, Van Rompaey KL, Michotte A, et al. Imaging tutorial: Differential diagnosis of bright lesions on diffusion-weighted MR images. Radiographics 2003;23:e7.

8. Tartaglino LM, Flanders AE, Vinitski S, Friedman DP. Metallic artifacts on MR images of the postoperative spine: Reduction with fast spin-echo techniques. Radiology 1994;190:565-9.

9. Poustchi-Amin M, Mirowitz SA, Brown JJ, McKinstry RC, Li T. Principles and applications of echo-planar imaging: A review for the general radiologist. Radiographics 2001;21:767-79.
10. Gann PH. Risk factors for prostate cancer. Rev Urol 2002;4 Suppl 5:S3-S10.

11. Tanimoto A, Nakashima J, Kohno H, Shinmoto H, Kuribayashi S. Prostate cancer screening: The clinical value of diffusion-weighted imaging and dynamic MR imaging in combination with T2-weighted imaging. J Magn Reson Imaging 2007;25:146-52.

12. Haider MA, van der Kwast TH, Tanguay J, Evans AJ, Hashmi AT, Lockwood G, et al. Combined T2-weighted and diffusion-weighted MRI for localization of prostate cancer. AJR Am J Roentgenol 2007;189:323-8.

13. Kozlowski P, Chang SD, Goldenberg SL. Diffusion-weighted MRI in prostate cancer-Comparison between single-shot fast spin echo and echo planar imaging sequences. Magn Reson Imaging 2008;26:72-6.

14. Outwater EK, Montilla-Soler JL. Imaging of prostate carcinoma. Cancer Control 2013;20:161-76.

15. Spencer JA, Chng WJ, Hudson E, Boon AP, Whelan P. Prostate specific antigen level and Gleason score in predicting the stage of newly diagnosed prostate cancer. Br J Radiol 1998;71:1130-5.

16. Singh P, Yadav MK, Singh SK, Lal A, Khandelwal N. Case series: Diffusion weighted MRI appearance in prostatic abscess. Indian J Radiol Imaging 2011;21:46-8.

17. Squillaci E, Manenti G, Mancino S, Carlani M, Di Roma M, Colangelo V, et al. MR spectroscopy of prostate cancer. Initial clinical experience. Journal of Experimental and Clinical Cancer Research: CR 2005;24:523-30.

18. Costouros NG, Coakley FV, Westphalen AC, Qayyum A, Yeh BM, Joe BN, et al. Diagnosis of prostate cancer in patients with an elevated prostate-specific antigen level: role of endorectal MRI and MR spectroscopic imaging. American Journal of Roentgenology 2007;188:812-6. 